LOW FREQUENCY CAPACITANCE MEASUREMENTS OF THE AGS BOOSTER ELECTROSTATIC PICK-UP ELECTRODES

BOOSTER TECHNICAL NOTE

NO. 178

D. J. CIARDULLO

SEPTEMBER 13, 1990

ALTERNATING GRADIENT SYNCHROTRON DEPARTMENT BROOKHAVEN NATIONAL LABORATORY UPTON, NEW YORK 11973 


\section{LOW FREQUENCY CAPACITANCE MEASUREMENTS OF THE AGS BOOSTER ELECTROSTATIC PICK-UP ELECTRODES}

\section{INTRODUCTION}

Capacitance measurements have been made on a typical Booster production PUE assembly. The detector is the split cylinder electrostatic type, which provides plate signals linearly related to the transverse position of the beam ${ }^{1}$. A fundamental difference between this and other split cylinder detectors, however, is the addition of a "calibration ring" which encircles both PUEs. The ring simulates a centered beam by coupling an applied input signal equally to both plates (this is useful for doing limited BPM system diagnostics without actually requiring beam). There is, however, an overall decrease in both the common mode and differential detector sensitivities, since the capacitances related to the calibration ring act parasitic to the electrodes. The detector and its associated capacitances are shown in Figure 1.

The purpose of this technical note is to obtain the value of each "isolated" capacitance element of the detector model shown in Figure 2:

$$
\begin{array}{lll}
\mathrm{C}_{\mathrm{pp}} & : & \text { Plate to plate capacitance } \\
\mathrm{C}_{\mathrm{pc}} & : & \text { Plate to calibration ring capacitance } \\
\mathrm{C}_{\mathrm{p}} & : & \text { Plate to detector shell capacitance } \\
\mathrm{C}_{\mathrm{c}} & : & \text { Calibration ring to shell capacitance } \\
\mathrm{C}_{\mathrm{f}} & : & \text { Vacuum feedthru N-type connector (to shell) }
\end{array}
$$

As can be seen from Figure 2, interactions due to coupling between the PUEs and the calibration ring make it difficult to measure any one isolated capacitance directly. However, a sufficient amount of information can be obtained by measuring specific element combinations. These "effective capacitances", described by the sub-models shown in Figures 3-5, can then be used to extract the isolated element values listed above. 


\section{EXTRACTION OF ISOLATED CAPACITANCES:}

On a completed detector assembly, the capacitance measurements which can be made directly are:

$\mathrm{C}_{\mathrm{P}}(\mathrm{eff}) \quad$ The effective PUE to shell capacity, looking into the electrode feedthru,

$\mathrm{C}_{\mathrm{C}}$ (eff) the effective calibration ring to shell capacity, looking into one of the calibration ring feedthrus, and

$\mathrm{C}_{\mathrm{Pp}}(\mathrm{eff})$ the effective inter-electrode capacity, as measured between the center conductors of both electrode vacuum feedthrus.

The term "effective" is used since each reading results from a different combination of all the capacitances within the detector (refer to Fig. 2).

These three measurement values provide insufficient information to completely determine the detector model of Figure 2. Additional measurements are needed which require partial disassembly of the detector.

\section{DETERMINING $\mathrm{C}_{\mathrm{f}}$}

First, mechanically separable components are removed from the assembly (elements in Figure 2 represented by dashed lines). These components include the vacuum feedthru connectors, as well as any external load on the calibration ring or the plates. Once isolated, the capacitance of each detached component is easily measured.

\section{DETERMINING $\mathrm{C}_{\mathrm{pp}}$ AND $\mathrm{C}_{\mathrm{pc}}$ :}

The second group of capacitance readings were taken on the subassembly formed by the split cylinder and the calibration ring outside of the detector shell (see figure 3). The measurements obtained from this subassembly are:

$$
\begin{gathered}
C_{p p}(e f f)=C_{p p}+\frac{C_{p c}}{2} \\
C_{p c}(e f f)=C_{p c}+\frac{C_{p c} C_{p p}}{C_{p c}+C_{p p}}
\end{gathered}
$$


where $C_{p p}(e f f)$ is the effective capacitance as measured across the plates (electrodes), and $\mathrm{C}_{\mathrm{pc}}(\mathrm{eff})$ is the effective capacitance from one plate to the calibration ring. Note that these values are the result of specific combinations of the capacitance elements shown in Figure 3 (lower case subscripts will be used to distinguish measurements made on subassemblies from those made on a completely assembled detector).

Equations (1) and (2) can be used to extract the element values for $\mathrm{C}_{\mathrm{pp}}$ and $\mathrm{C}_{\mathrm{pc}}$ from their measured "effective" capacitances:

$$
\begin{gathered}
C_{p p}=C_{p p}(e f f)\left[1-\frac{C_{p c}(e f f)}{4 C_{p p}(e f f)-C_{p c}(e f f)}\right] \\
C_{p c}=2 \frac{C_{p p}(e f f) C_{p c}(e f f)}{4 C_{p p}(e f f)-C_{p c}(e f f)}
\end{gathered}
$$

\section{DETERMINING $\mathrm{C}_{\mathrm{p}}$ AND $\mathrm{C}_{\mathrm{c}}$ :}

The last group of readings are taken on the detector assembly, less any vacuum feedthru connectors (or other external load). The measurements obtained from this assembly are (refer to Figures 4 and 5):

$$
\begin{gathered}
C_{p}(e f f)=C_{p}+\frac{C_{p c} C_{c}}{C_{c}+2 C_{p c}}+\frac{1}{\frac{1}{C_{p p}+\frac{C_{p c}^{2}}{C_{c}+2 C_{p c}}}+\frac{1}{C_{p}+\frac{C_{p c} C_{c}}{C_{c}+2 C_{p c}}}} \\
C_{c}(e f f)=C_{c}+\frac{C_{p c} C_{p}}{C_{p}+C_{p p}+C_{p c}}+\frac{1}{\frac{1}{C_{p c}+\frac{C_{p c} C_{p p}}{C_{p}+C_{p p}+C_{p c}}}+\frac{1}{C_{p}+\frac{C_{p} C_{p p}}{C_{p}+C_{p p}+C_{p c}}}}
\end{gathered}
$$

where $C_{p p}$ and $C_{p c}$ are calculated from equations (3) and (4). Figures 4 and 5 illustrate the capacitive elements which make up $C_{p}\left(\right.$ eff) and $C_{c}(e f f)$, respectively. Note that "wyedelta" impedance transformations are used to simplify both submodels. The general wyedelta transformation is shown in Appendix $1 .^{3}$ 
Values for $C_{p}$ and $C_{c}$ have been extracted from equations (5) and (6) using the program MACSYMA. Appendix 2 shows the solution for $C_{p}$ in terms of $C_{p p}, C_{p c}$ and the measured "effective" capacitances. Once $C_{p}$ has been determined, eq. (6) is used to solve for the calibration ring capacitance, $\mathrm{C}_{\mathrm{c}}$.

\section{ACTUAL MEASUREMENT VALUES:}

The following effective capacitance readings were taken on a "typical" production electrostatic detector (S/N 039):

$\begin{array}{lll}\mathrm{C}_{\mathrm{f}} & : & 7 \mathrm{pF} \\ \mathrm{C}_{\mathrm{pp}}(\mathrm{eff}) & : & 24 \mathrm{pF} \\ \mathrm{C}_{\mathrm{pc}}(\mathrm{eff}) & : & 17 \mathrm{pF} \\ \mathrm{C}_{\mathrm{p}}(\mathrm{eff}) & : & 45 \mathrm{pF} \\ \mathrm{C}_{\mathrm{c}}(\mathrm{eff}) & : & 56 \mathrm{pF}\end{array}$

These measurements were taken with an HP4262A LCR meter. $C_{f}$ is an actual element in the detector model of Figure 2; the remaining components in the model can now be calculated using eq. (3), (4), (6) and Appendix 2. The following values result:

$\begin{array}{lll}\mathrm{C}_{\mathrm{pp}} & = & 18.8 \mathrm{pF} \\ \mathrm{C}_{\mathrm{pc}} & = & 10.3 \mathrm{pF} \\ \mathrm{C}_{\mathrm{p}} & = & 25.6 \mathrm{pF} \\ \mathrm{C}_{\mathrm{c}} & = & 41.3 \mathrm{pF} \\ \mathrm{C}_{\mathrm{f}} & = & 7 \mathrm{pF}\end{array}$

(by direct measurement)

Figure 6 shows the capacitance model for the typical Booster average orbit electrostatic detector, with the actual element values listed. Note that the external load capacitance on each PUE $\left(C_{L}(p)\right)$ depends on the BPM system gain, and can take one of two values $(210 \mathrm{pF}$ or $2610 \mathrm{pF})$. $\mathrm{C}_{\mathrm{L}}(\mathrm{c})$ is the capacitance loading due to the cable assembly connecting the calibration ring to the BPM electronics (approximately $285 \mathrm{pF}$ at low frequencies).

\section{EFFECTIVE PUE CAPACITANCE LOAD:}

The effective electrode capacitance, $C_{\text {pue }}(\mathrm{eff})$, is the load that the beam "sees" due to each PUE. This is defined by equation (7) below, which is equation (5) with the following modifications:

$$
\begin{aligned}
& . C_{p} \text { replaced by } C_{p}+C_{f}+C_{L}(p) \\
& . C_{c} \text { replaced by } C_{c}+2 C_{f}+C_{L}(c)
\end{aligned}
$$

These modifications are necessary to account for the capacitance of the vacuum feedthru connectors, as well as any external loading of the detector. 
Capacitance the beam "sees" from each PUE:

$$
C_{p u e}(e f f)=C_{p}^{\prime}+\frac{C_{p c} C_{c}^{\prime}}{C_{c}^{\prime}+2 C_{p c}}+\frac{1}{C_{p p}+\frac{C_{p c^{*}}^{2}}{C_{c}^{\prime}+2 C_{p c}}}+\frac{1}{C_{p}^{\prime}+\frac{C_{p c} C_{c}^{\prime}}{C_{c}^{\prime}+2 C_{p c}}}(7)
$$

where

$$
\begin{aligned}
& C^{\prime}=C_{p}+C_{f}+C_{L}(p) \\
& C_{c}^{p}=C_{c}+2 C_{f}+C_{L}(c)
\end{aligned}
$$

\section{CONCLUDING REMARKS}

A capacitance model of the Booster average orbit electrostatic detector has been presented in Figure 6. The values of the individual capacitive elements have been calculated from measurements made on specific detector subassemblies.

Equation (7) gives the effective capacitance load presented to the beam from each electrode. It includes the effects of the vacuum feedthrus, the loading of the BPM electronics front end, and the capacitance due to the system connecting cables.

It is interesting to note that large changes in the capacitance loading of the calibration ring have only a small effect on the value of $\mathrm{C}_{\text {pue }}$ (eff). At low frequencies, this effect is reduced even further when the calibration ring is loaded with 50 ohms (after installation into the Booster). The $\mathbf{5 0} \mathrm{ohm}$ load bleeds off any charge trying to accumulate on $\mathrm{C}^{\prime}$ (i.e., the cal ring, its connecting cable and two vacuum feedthrus). This essentially eliminates the capacitance contribution from $\mathrm{C}^{\cdot}{ }_{\mathrm{c}}$ and equation (7) can be approximated by: 


$$
\begin{gathered}
C_{p \text { us }}(\text { eff })-C_{p}^{\prime}+\frac{1}{\frac{1}{C_{p p}+\frac{C_{p c}}{2}}+\frac{1}{C_{p}^{\prime}}} \\
=C_{p}^{\prime}+\frac{1}{\frac{1}{24}+\frac{1}{C_{p}{ }^{\prime}}}
\end{gathered}
$$

$$
\begin{aligned}
& \text { with } \mathrm{C}_{\mathrm{p}}{ }^{\circ}=\mathrm{C}_{\mathrm{p}}+\mathrm{C}_{\mathrm{f}}+\mathrm{C}_{\text {cable }}+\mathrm{C}_{\text {fep }} \\
& =25.6+7+153+50 \text { (x1, x10 BPM system gains) } \\
& 2450 \text { (x0.1 BPM system gain) } \\
& \text { (all values in } \mathrm{pF} \text { ) }
\end{aligned}
$$

Using equation (9), the calibration ring is found to contribute an increase of $\mathrm{C}_{\text {pue }}$ (eff) of less than $2 \%$ for the X1 and X10 BPM system gain modes, and less than $0.2 \%$ for the $\mathrm{x} 0.1$ gain mode.

\section{ACKNOWLEDGEMENT:}

The assistance of R. Thomas in both the use of MACSYMA and the review of this technical note is gratefully acknowledged. In addition, the support given by $\mathrm{J}$. Cupolo in making the actual detector measurements is much appreciated.

\section{REFERENCES:}

1. R. E. Shafer, Beam Position Monitoring, Brookhaven Instrumentation Workshop (1989).

2. G. R. Lambertson, "Electromagnetic Detectors", in Frontiers of Particle Beams; Observation, Diagnosis and Correction (M. Month, S. Turner, eds.), p.380. SpringerVerlag, New York (1989).

3. W. H. Hayt, Jr., J. E. Kemmerly, Engineering Circuit Analysis, third edition, New York: McGraw Hill, 1978. 


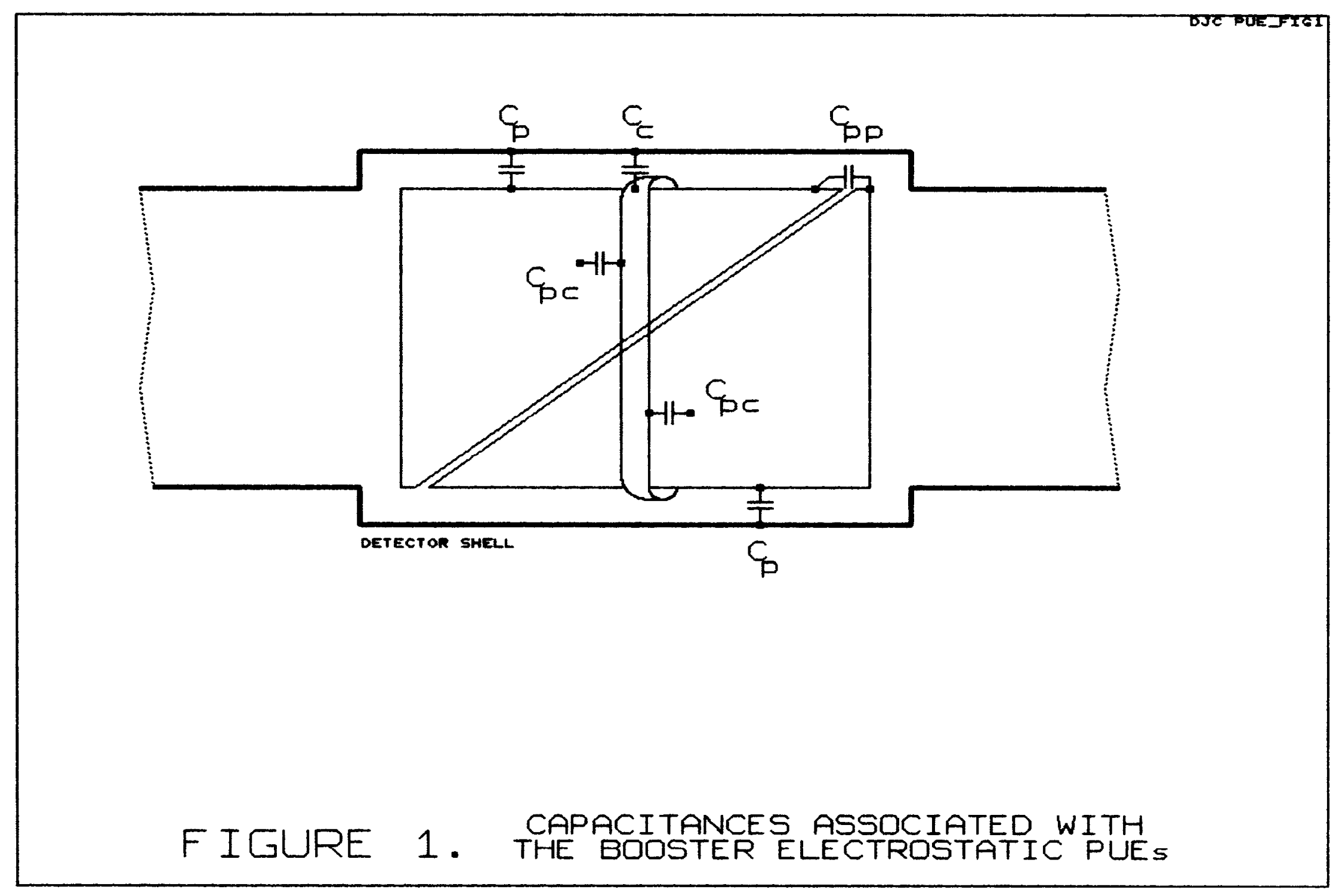









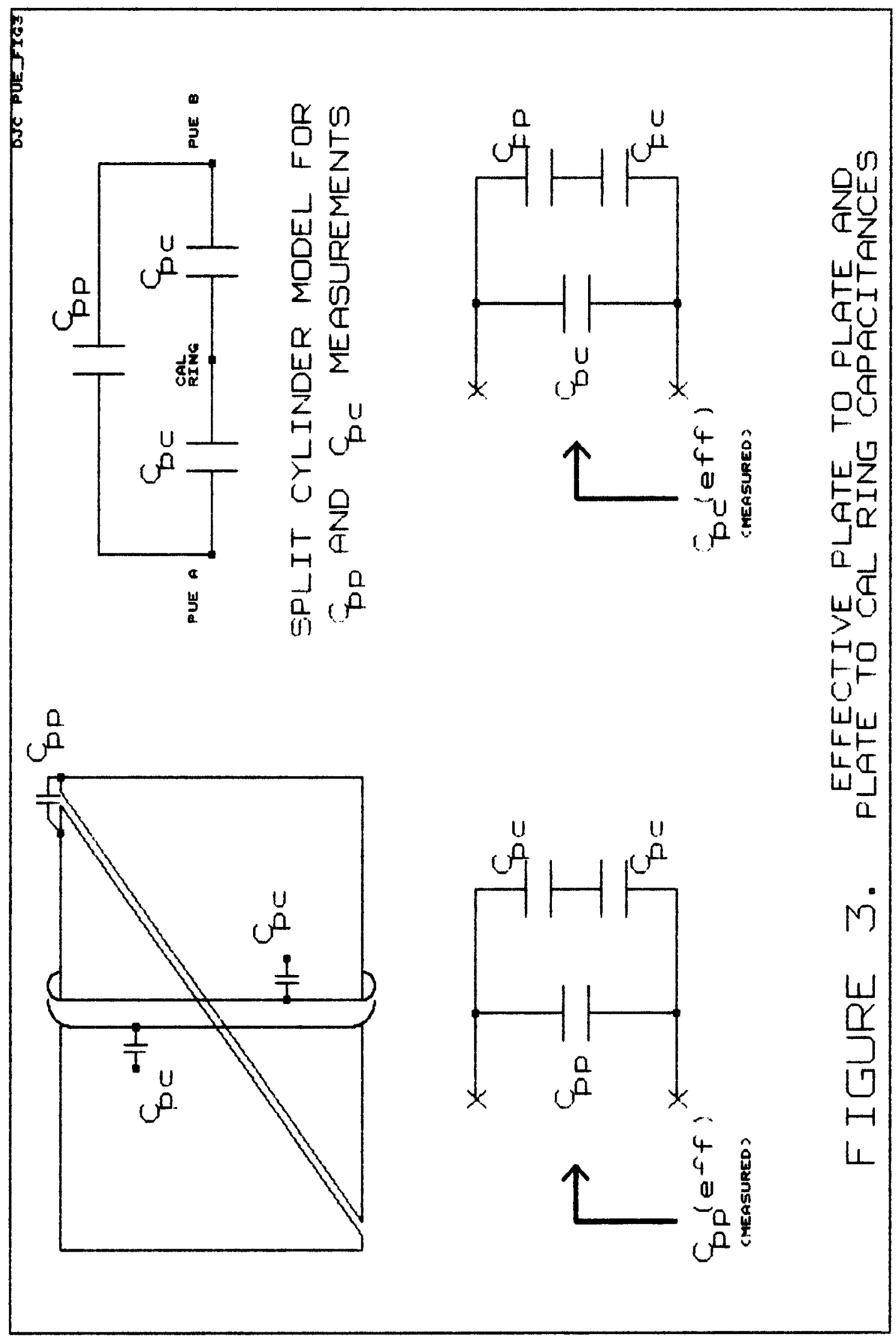




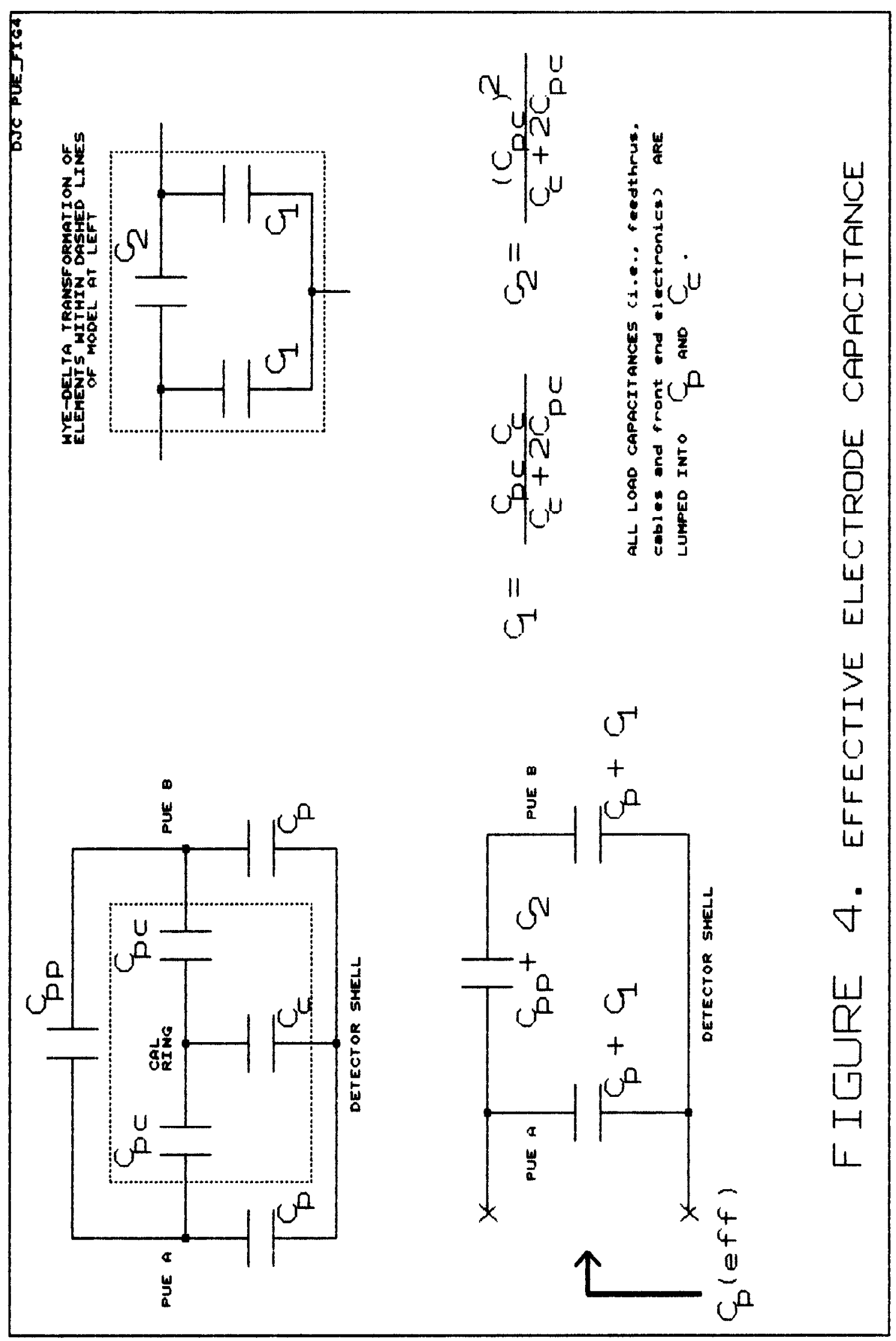




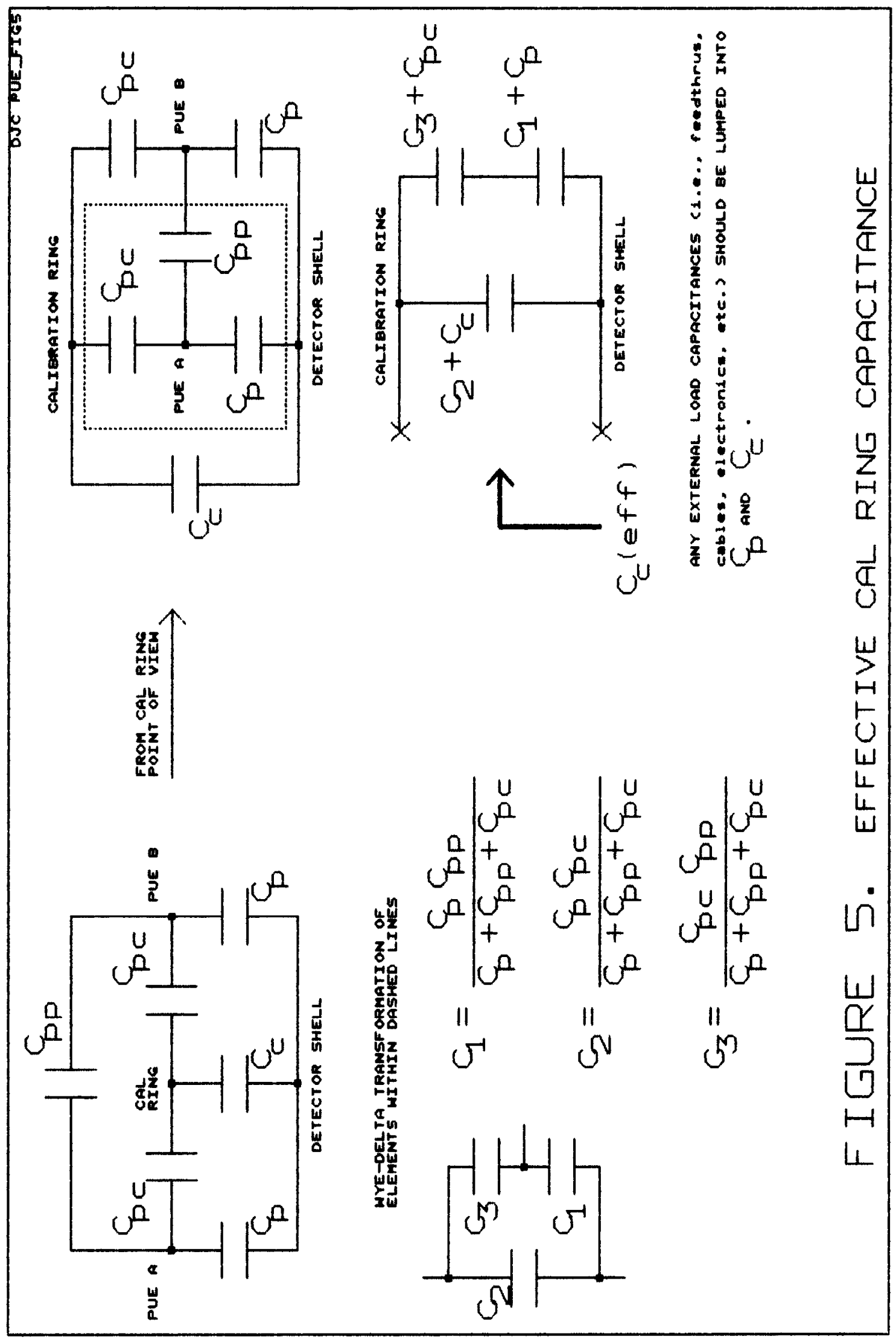




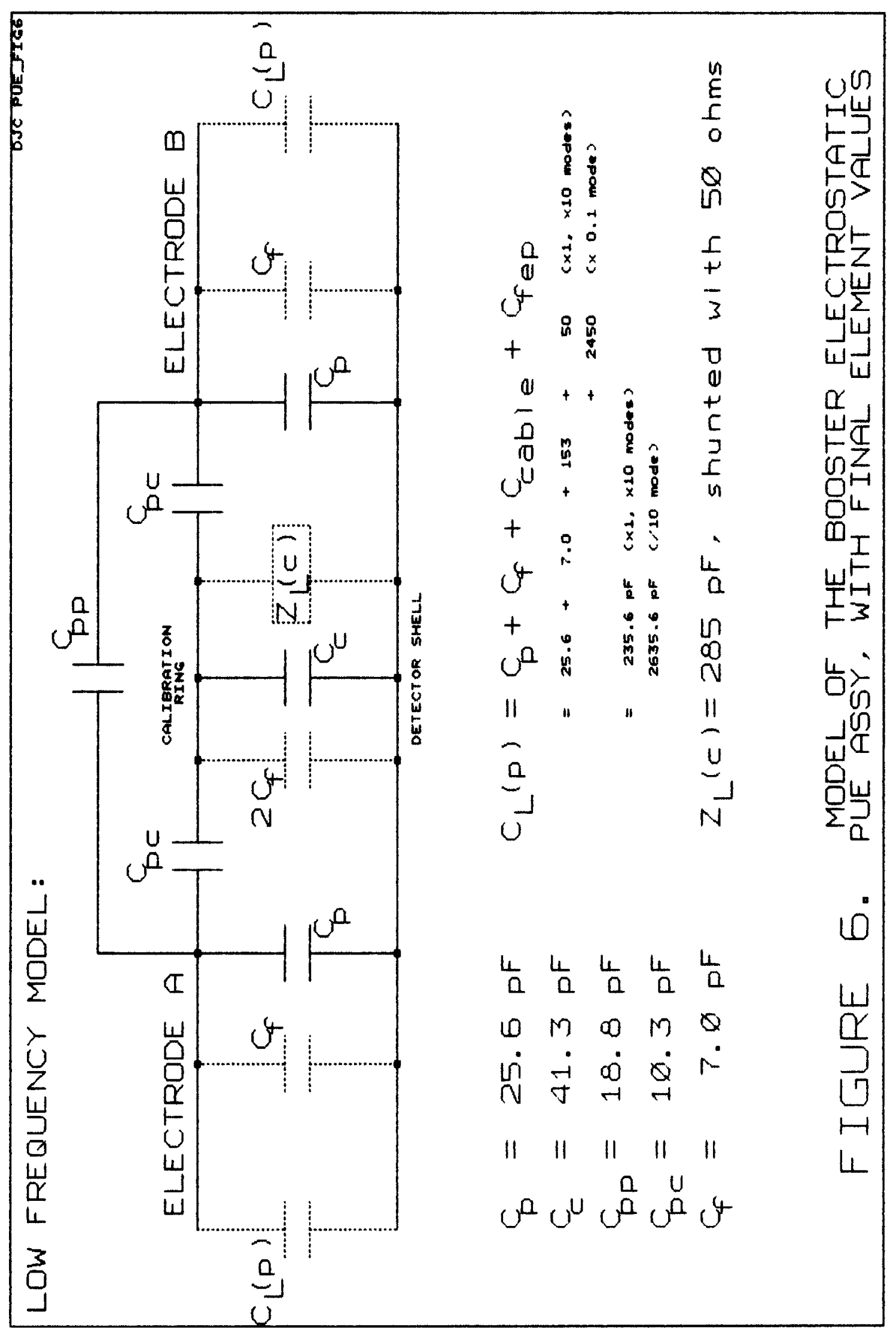









\section{APPENDIX 2 \\ SOLUTION FOR $C_{P}$ :}

$\mathrm{cp}=\operatorname{expt}\left(\operatorname{sqrt}\left(-\operatorname{cpeff}\left[\left(4 \operatorname{cceff}^{3} \operatorname{cpeff}+64 \operatorname{cceff}^{2} \operatorname{cpc}^{2}\right) \operatorname{cpp}^{4}\right.\right.\right.$

$-16 \operatorname{cceff}^{2} \mathrm{cpc}^{2}$ cpeff $\mathrm{cpp}^{3}+\operatorname{cceff}^{3} \operatorname{cpeff}^{3}+16 \operatorname{cceff}^{2} \mathrm{cpc}^{2} \operatorname{cpeff}^{2}$

-32 cceff $c p c^{4}$ cpeff) $\mathrm{cpp}^{2}+\left(-6 \operatorname{cceff}^{2} \mathrm{cpc}^{2} \operatorname{cpeff}^{3}-28 \operatorname{cceff} \operatorname{cpc}^{4} \operatorname{cpeff}^{2}\right)$ $\left.\left.\mathrm{cpp}+\operatorname{cceff} \mathrm{cpc}^{4} \operatorname{cpeff}^{3}+4 \operatorname{cpc}^{6} \operatorname{cpeff}^{2}\right] / \operatorname{cceff}\right) /(6 \operatorname{sqrt}(3) \operatorname{cceff})$

$+\left(\operatorname{cceff}\left(-16 \mathrm{cpp}^{3}+6\right.\right.$ cpeff cpp $\left.{ }^{2}-3 \operatorname{cpeff}^{2} \mathrm{cpp}+2 \operatorname{cpeff}^{3}\right)$

$+\operatorname{cpc}^{2}\left(36\right.$ cpeff cpp $\left.\left.+9 \operatorname{cpeff}^{2}\right)\right) /(54$ cceff $), \begin{gathered}1 \\ -1 \\ 3\end{gathered}$

$+\left(\operatorname{cceff}\left(4 \mathrm{cpp}^{2}-\operatorname{cpeff} \mathrm{cpp}+\operatorname{cpeff}^{2}\right)+3 \operatorname{cpc}^{2} \operatorname{cpeff}\right)$

$/\left(9\right.$ cceff expt (sqrt $\left(-\operatorname{cpeff}\left(\left(4 \operatorname{cceff}^{3}\right.\right.\right.$ cpeff $\left.+64 \operatorname{cceff}^{2} \operatorname{cpc}^{2}\right) \operatorname{cpp}^{4}$

$-16 \operatorname{cceff}^{2} \mathrm{cpc}^{2} \operatorname{cpcff} \mathrm{cpp}^{3}+\operatorname{cceff}^{3} \operatorname{cpeff}^{3}+16 \operatorname{cceff}^{2} \operatorname{cpc}^{2} \operatorname{cpeff}^{2}$

-32 cceff $\operatorname{cpc}^{4}$ cpeff) $\operatorname{cpp}^{2}+\left(-6 \operatorname{cceff}^{2} \operatorname{cpc}^{2} \operatorname{cpeff}^{3}-28 \operatorname{cceff} \operatorname{cpc}^{4} \operatorname{cpeff}^{2}\right)$ $\left.\left.\operatorname{cpp}+\operatorname{cceff} \operatorname{cpc}^{4} \operatorname{cpeff}^{3}+4 \operatorname{cpc}^{6} \operatorname{cpeff}^{2}\right) / \operatorname{ceff}\right) /(6 \operatorname{sqrt}(3) \operatorname{cceff})$

$+\left(\operatorname{cceff}\left(-16 \mathrm{cpp}^{3}+6 \operatorname{cpeff}_{\mathrm{cpp}}{ }^{2}-3 \operatorname{cpeff}^{2} \mathrm{cpp}+2 \operatorname{cpeff}^{3}\right)\right.$

$+\operatorname{cpc}^{2}\left(36\right.$ cpeff $\left.\left.\operatorname{cpp}+9 \operatorname{cpeff}^{2}\right)\right) /\left(54\right.$ cceff),$\left.\left.\frac{1}{3}\right)\right)-\frac{2 \mathrm{cpp}-\mathrm{cpeff}+3 \mathrm{cpc}}{3}$ 